\title{
LOS EFECTOS DE LAS ANTOCIANINAS EXTRAÍDAS DE Zea mays L. (MAÍZ MORADO) SOBRE LAS HIPERLIPIDEMIAS EN RATAS ALBINAS
}

\author{
Enzio Foy Valencia
}

\begin{abstract}
RESUMEN
El propósito del presente estudio fue determinar el efecto de una dieta suplementada con antocianinas extraídas de la coronta de Zea mays L. (maíz amiláceo morado) sobre la concentración de lípidos y lipoproteínas séricas en ratas albinas. El diseño experimental consistió en extraer el pigmento por medio del proceso de atomización y extracto acuoso hervido conocido como chicha morada. Se trabajó con 12 ratas albinas en condiciones de laboratorio midiendóse los lípidos basales, luego se les indujo a una hiperlipidemia consumiendo por 15 días una dieta hiper-grasa. Luego de verificar mediante una toma de muestra sanguínea que el perfil lipídico se incrementó (triglicéridos, colesterol y lipoproteínas de baja densidad LDL “colesterol malo") se procedió a proporcionarles las antocianinas como un suplemento de su dieta; en polvo al 5\% y extracto acuoso (chicha morada) al 20\% durante 15 días. Al término del cual se determinó nuevamente el perfil lipídico. Lográndose una reducción del 79.40\% en triglicérido, colesterol total disminuyó en 66,54\% y lipoproteínas de baja densidad LDL “colesterol malo” disminuyó en 90,74\%.
\end{abstract}

Palabras claves: Hyperlipidemia, LDL, Anthocyanins.

\section{SUMMARY}

The purpose of this study was to determine the effect of the diet supplemented with anthocyanin extracts from the Zea mays L. (purple maize) on the concentration of lipids and blood lipoproteins in albino rats. The experimental design consisted of extracting the pigment by the process of atomization and boiled aqueous extract known as chicha morada. We worked with 12 albino rats under laboratory conditions lipids measured at baseline, then were induced hyperlipidemia eating a 15-day hyper-fat diet. After checking by taking a blood sample that increased lipid profile (triglycerides, cholesterol and low density lipoprotein LDL "bad cholesterol") proceeded to provide the anthocyanins as a dietary supplement, $5 \%$ powder and extract aqueous (chicha morada) to $20 \%$ for 15 days. After which it again found the lipid profile. Achieving a $79.40 \%$ reduction in triglycerides, total cholesterol decreased by $66.54 \%$ and low density lipoprotein LDL "bad" cholesterol decreased by $90.74 \%$.

Key Words: Hyperlipidemia, LDL, Anthocyanins.

\section{INTRODUCCIÓN}

En la actualidad se ha incrementado el consumo de alimentos elaborados con contenidos altos de lípidos que ocasionan enfermedades al sistema cardiovascular en el hombre; de allí que se busca alimentos o productos naturales que puedan solucionar dichos problemas de salud sin los efectos colaterales que pueden ocasionar el uso de productos o medicamentos sintéticos o no naturales; siendo así que, el maíz morado y sus pigmentos constituyen un alimento promisorio para el mejoramiento de la salud humana.

El maíz (Zea mays L. Amiláceo) es una gramínea, originaria del continente americano. Para el caso del maíz amiláceo morado se encuentra en el área andina de Bolivia, Chile, Argentina, Ecuador y Perú.
Se consume como grano sancochado, harina y preparación de bebidas. En el caso de maíz amiláceo morado, se le emplea para la obtención de pigmentos, en especial la antocianina que se utiliza como colorante de alimentos, cosméticos, textiles y pinturas.

La materia colorante del maíz morado son las antocianinas de las cuales se han determinado, 3-glucósidos de cianidina, pelargonidina y peonidina, 3-galactósido de cianidina, libres y acilado. También nos dice que actualmente se preparan extractos acuosos atomizados; sin embargo, los volúmenes de producción aún no compiten con las enocininas. (Solano, Ricardo. 1999).

Fernández (1995) estudió en una primera etapa la influencia de los parámetros de maceración de la coronta y la combinación de maceración y calentamiento en 
el rendimiento de la extracción de antocianinas. En una segunda etapa estudió la purificación del extracto mediante precipitación con acetato de plomo básico y resinas de intercambio iónico.

En lo referente a las antocianinas del maíz morado, estas son colorantes naturales obtenidos a partir del maíz morado, una variedad única que se cultiva en el Perú, y viene siendo utilizada durante cientos de años para elaborar bebidas y postres. Sus coloraciones van del morado rojizo al morado muy oscuro. Se utiliza en bebidas, vegetales en conserva, mermeladas, dulces, panificación, helados, gelatinas, gomas de mascar, salsas, etc. La Antocianina, motivo de los estudios, se concentra principalmente en la coronta del maíz morado y se exporta desde el Perú hacia países como Japón y Alemania que lo utilizan para " balancear " el color de los licores de fantasía. A nivel local se emplea la antocianina para colorear yogur (Salinas Moreno, et al. .2005) y en menores cantidades para bebidas alcohólicas, especialmente vinos.

El efecto de las antocianinas fue probado en conejos diabéticos inducidos con aloxano y luego de 28 días de observación se verificó experimentalmente que la antocianina fue más eficaz, en inducir la caída de los niveles de glucosa, con reducciones de 10,78 por ciento a los 7 días, 10,54 por ciento a los 14 días, 17,33 por ciento a los 21 días. Se observó reducción de los porcentajes de variación de triglicéridos en 22,74 por ciento por la antocianina y 22,60 por ciento por el tratamiento con própolis a los 28 días (Oliveira, Tânia y cols. 2002)

La antocianina, de fuerte presencia en el maíz morado, parece ser una de las sustancias más saludables para combatir el envejecimiento. La antocianina es un flavonoide, o un antioxidante de la planta. Los antioxidantes poseen la capacidad de neutralizar el efecto de los radicales libres. Estas moléculas inestables, los radicales libres, son capaces de atacar las células del organismo y dañar su ADN, siendo la causa del cáncer y las enfermedades relacionadas con el envejecimiento. También, ayuda a que la piel se regenere evitando arrugas de la piel (Hernández, Humberto 2003)

El objetivo de la presente investigación es determinar el efecto de una dieta suplementada con antocianinas extraídas de la coronta de Zea mays L. (maíz amiláceo morado) sobre la concentración de lípidos y lipoproteínas séricas en ratas albinas.

\section{MATERIAL Y MÉTODOS}

\section{Extracción de las antocianinas por atomización}

Se uso el flujo general de operaciones para la obtención de antocianinas del maíz morado, de acuerdo a la Figura 1. El extracto fue obtenido del maíz morado procedente de la provincia de Canta, Dpto. de Lima, a partir de la coronta del maíz en una proporción del 20\% bajo la forma de chicha morada, la cual fue administrada como bebida ad libitum a los animales de experimentación.

Las antocianinas fueron extraídas por atomización, luego por calentamiento a $100^{\circ} \mathrm{C}$. que es la forma común de preparación de la chicha morada.

\section{Diseño experimental}

Se preparo una dieta suplementada con antocianinas extraídas por atomización de la coronta de Zea mays $L$. (maíz amiláceo morado) al 5\% y otra con el extracto de coronta de maíz morado hervido al $20 \%$, en el lapso de dos semanas

Se trabajó con dos bloques de especimenes de ratas albinas machos, uno para el consumo de dieta con $5 \%$ de antocianinas y otro para la utilización del extracto de maíz morado hervido al $20 \%$ o chicha morada; es decir, una dieta suplementada con antocianinas extraídas de la coronta de Zea mays L. (maíz amiláceo morado) y la otra suplementada con extracto hervido del maíz. Con un total de 12 ratas albinas divididas en dos bloques experimentales.

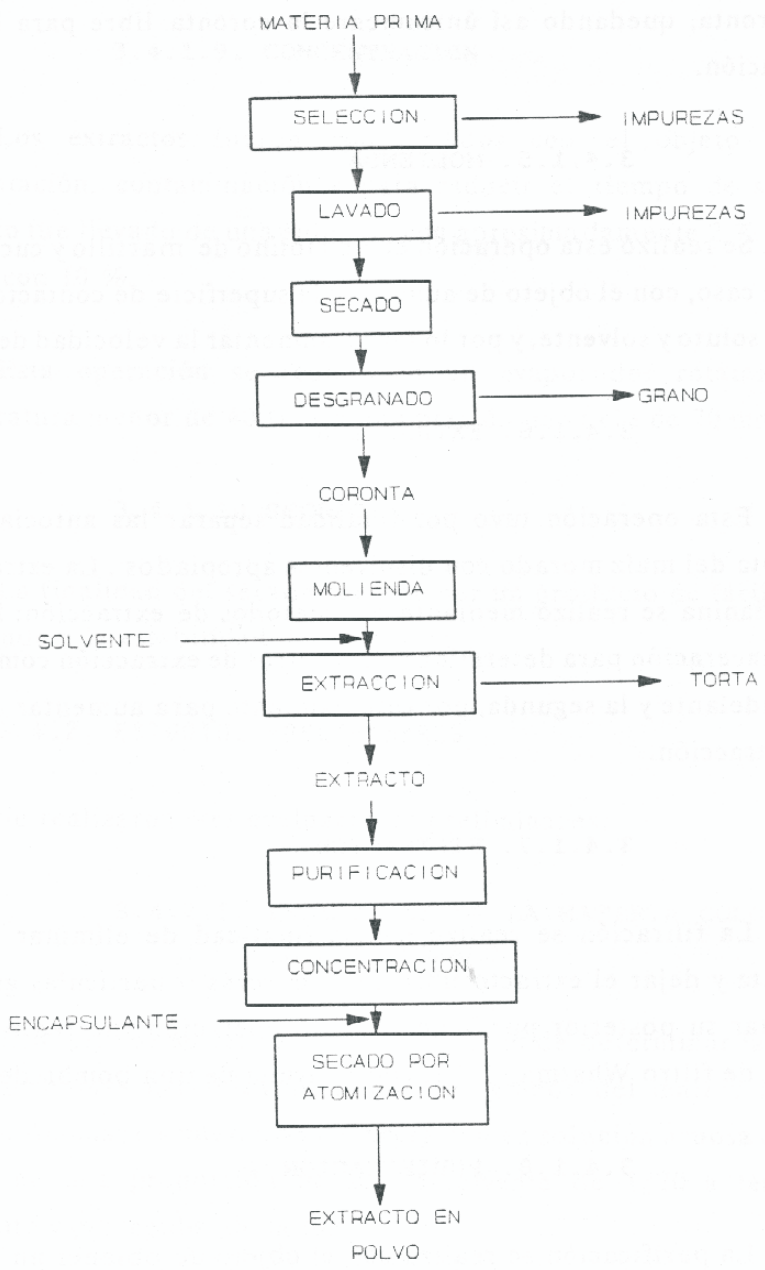

Figura 1. Flujograma de extracción de antocianinas del maíz morado. 
La dieta basal que se le administró a los dos bloques consistió de una dieta libre de colesterol, la cual estuvo conformada por insumos de origen vegetal, proporcionada a las ratas por una semana a fin de adecuarlas a las condiciones de crianza del bioterio.Aquí se tomó una primera muestra sanguínea para verificar las concentraciones basales de lípidos

A los dos bloques experimentales se le indujo a una hiperlipidemia, administrándose una dieta con alimentos grasos de uso común (se le dio de forma alternada pizzas de queso con embutidos, así como yema de huevos de gallina e hígado de pollo hervidos), por el periodo de tres semanas. Al término de las cuales se les tomó a las ratas una segunda muestra de sangre con la finalidad de verificar la elevación de las concentraciones de lípidos sanguíneos.

Posteriormente a los animales se les separó en dos grupos experimentales. A uno de los grupos que se le indujo a la hiperlipidemia se le administró una dieta suplementada con antocianinas al 5\% extraídas de la coronta de Zea mays L. (maíz amiláceo morado) por un lapso de dos semanas y a un segundo grupo que se le indujo a la hiperlipidemia se le adicionó en la dieta extracto hervido de la coronta de Zea mays L. al 20 \% (maíz amiláceo morado) (chicha morada) por un lapso de dos semanas.

Al cabo de haberse administrado la dieta suplementada con antocianinas extraídas de la coronta de Zea mays $L$. (maíz amiláceo morado) y adicionado la chicha morada en la dieta por dos semanas también; se realizó un tercer análisis bioquímico del suero sanguíneo para comprobar el efecto sobre los niveles de lípidos del producto que estamos investigando.

Para el tratamiento de la dieta con grasa animal, como para la dieta suplementada con antocianinas extraídas de la coronta de Zea mays L. (maíz amiláceo morado), el periodo de aplicación fue por un lapso de tres y dos semanas respectivamente.

Se determinó los niveles séricos de colesterol, triglicéridos, LDL colesterol por métodos enzimáticos.

Al término del experimento los animales fueron sacrificados.

\section{RESULTADOS}

Los resultados se pueden apreciar en las tablas 1, 2, 3, $4, \mathrm{y} 5$.

\section{DISCUSIÓN}

Los triglicéridos sanguíneos se reducen notablemente $(79,40 \%)$ cuando se realiza la ingestión de una dieta suplementada con extractos de antocianinas presentes en el maíz morado, ya sea ingiriéndolos como componentes de la dieta en una proporción al $5 \%$ o bebiéndola en una proporción al 20\% (como chicha morada). Considerando que los niveles de triglicéridos fueron incrementados por ingestión de una dieta excesiva de grasa (dieta hipergrasa), al incluirse en la dieta los pigmentos del maíz morado o antocianinas los niveles de triglicéridos disminuyeron. Si tenemos en cuenta que una elevación de los triglicéridos sanguíneos es perjudicial para la salud de las personas debemos considerar que el consumo de los pigmentos en cualquiera de sus formas (como pigmento o como extracto) podría ayudar a una reducción de sus valores sanguíneos y por consiguiente a mejorar su salud con respecto a las concentraciones de lípidos.

Con respecto al colesterol total sanguíneo, éste es un componente de los lípidos corporales que cuando se elevan sus concentraciones sanguíneas trae problemas cardiovasculares y cerebro vasculares que pueden poner en peligro la salud de las personas, por consiguiente, el hecho que al haber inducido de forma experimental a una elevación del colesterol sanguíneo (hipercolesterolemia) en las ratas albinas durante 15 días, para luego hacerles un tratamiento con las antocianinas (los pigmentos del maíz morado) por 15 días también, permitieron una reducción bastante apreciable $(66,54 \%)$ de sus concentraciones sanguíneas. Por lo tanto, consideramos que si una persona que presenta elevaciones de su colesterol total, se somete a un tratamiento en el cual consuma como suplemento dietético las antocianinas (sea como pigmento o como extracto, es decir la chicha morada) podrá lograr una reducción y un mejoramiento de su colesterolemia. Es importante destacar que consumiendo tanto el pigmento como la chicha morada se logran reducciones en proporciones similares en las concentraciones del colesterol, lo cual significa que en esencia son los pigmentos extraídos los cuales logran las referidas disminuciones. Si tenemos en cuenta que cuando el colesterol se encuentra elevado se puede generar una ateroesclerosis (endurecimiento de las arterias y una posterior obstrucción arterial) que por lo general es de carácter irreversible; entonces consumiendo las antocianinas del maíz morado se puede lograr una prevención y evitar que se generen las alteraciones referidas o en todo caso evitar que éstas se agraven en caso que ya existan.

Los lípidos o grasas son compuestos no solubles en agua, entonces para ser transportados en la sangre se forman unos compuestos denominados lipoproteínas que permiten transportarlos por la circulación general; éstas lipoproteínas pueden ser de diversos tipos según su densidad, una de ellas son las lipoproteínas de baja densidad (conocidas como LDL) que se caracteriza por presentar en su composición una alta concentración de colesterol que se va quedando en la circulación y en las arterias y que cuando sus concentraciones se elevan traen los problemas de endurecimiento de las arterias y sus consiguientes problemas cardiovasculares y cerebro vascula- 
res; razón por la cual también se le conoce como el "colesterol malo". En el diseño experimental al proporcionarles a las ratas albinas la dieta hipergrasa durante 15 días se logró una elevación de estas lipoproteínas LDL ("colesterol malo"), y que cuando a los referidos animales de laboratorio, se les sometió al tratamiento con las antocianinas se logró una reducción del 90,74\%, lo cual consideramos que es un valor sorprendente en lo referente a una disminución del colesterol considerado como "malo" el cual es sumamente perjudicial para nuestro organismo. Como resultado de nuestro estudio estamos en condiciones de poder afirmar que el consumo de las antocianinas del maíz morado mejoran los niveles de las lipoproteínas de baja densidad LDL o “colesterol malo" y podrían servir para la prevención de los problemas circulatorios ocasionados por las elevaciones de estas fracciones de los lípidos sanguíneos.

Los resultados obtenidos se pueden proyectar hacia las personas en razón que el metabolismo de lípidos en las ratas albinas responde de manera similar a como sucede con los seres humanos, de allí que los trabajos experimentales de este tipo se suelen realizar con estos animales de laboratorio.

\section{CONCLUSIONES}

- El consumo de extracto de antocianinas del maíz morado en una proporción del $20 \%$ reduce los niveles de triglicéridos desde 180,8 mg/dL hasta 48,17 $\mathrm{mg} / \mathrm{dL}$ es decir un 73,4\%.

- El consumo de extracto antocianinas del maíz morado en una proporción del $20 \%$ reduce los niveles de colesterol desde $96,66 \mathrm{mg} / \mathrm{dL}$ hasta $35,35 \mathrm{mg} / \mathrm{dL}$ lo cual significa una reducción del 63,39\%.

- El consumo de extracto de antocianinas maíz morado en una proporción del $20 \%$ reduce los niveles de colesterol LDL (colesterol malo) desde 23,62 $\mathrm{mg} / \mathrm{dL}$ hasta $3,08 \mathrm{mg} / \mathrm{dL}$, y representa una disminución del 86,96\%.

- El consumo de los pigmentos antocianinas extraídos del maíz morado y consumidos en una proporción del 5\% reduce los niveles de triglicéridos desde $180,6 \mathrm{mg} / \mathrm{dL}$ hasta $26,36 \mathrm{mg} / \mathrm{dL}$ reduciéndolos en $85,41 \%$.

- El consumo de los pigmentos antocianinas extraídos del maíz morado y consumidos en una proporción del $5 \%$ reduce los niveles de colesterol desde $96,43 \mathrm{mg} / \mathrm{dL}$ hasta $29,22 \mathrm{mg} / \mathrm{dL}$ lo cual significa una disminución de $69,69 \%$.

- El consumo de los pigmentos antocianinas extraídos del maíz morado y consumidos en una proporción del 5\% reduce los niveles de colesterol LDL (colesterol malo) desde 23,39 mg/dL hasta 1,28 mg/dL disminuyéndolo en $94,52 \%$.

\section{LITERATURA CITADA}

CEDEP. 1999. Una introducción al cultivo de ají páprika. Revista cedep. 15 pp.

DELGADO E. J. 1989. Ensayos sobre el uso de microencapsulantes en el secado por atomización de concentrado de maíz morado (Zea mays L.). Tesis UNA. Lima.

FERNÁNDEZ, N. A. 1995. Estudio de la extracción y pre purificación de antocianinas de maíz morado (Zea mays L.). Tesis. UNA. Lima

FERNÁNDEZ N.A 1995. Estudio de la extracción y pre purificación de antocianinas de maíz morado (Zea mays L.). Tesis UNA. Lima.

FUENTES WL, IPARRAGUIRRE FH Y DELGADO R. 2004. Efecto del refresco de maíz morado y vino tinto en la agregación plaquetaria en personas sanas. Anales de la Facultad de Medicina. In press.

IPARRAGUIRRE FH, Fuentes WL 2004. Efecto del concentrado liofilizado de Zea mays morado en la inhibición de la agregación plaquetaria en conejos. Anales de la Facultad de Medicina In press.

IPARRAGUIRRE FH, FUENTES Wl. 2004. Efecto del concentrado liofilizado de Zea mays morado en la inhibición de la agregación plaquetaria en voluntarios sanos. Anales de la Facultad de Medicina. En prensa.

GIACONI, V. 1990. Cultivo de hortalizas. ed. universitarias. santiago-chile. 308 pp.

GOULD, K.S. Y LEE D.W. 2002. Antricyanins in Leaves. Advances in Botanical Research, Editores Vol. 37, Octubre.

HERNÁNDEZ SÁNCHEZ, HUMBERTO, 2003. Antioxidantes en alimentos. Universidad Autónoma de Nuevo León Revista de la Facultad de Salud Pública y Nutrición Vol. 4 No.4 OctubreDiciembre

MAROTO, J. 1986. Horticultura herbacea y especial. ed. mundi-prensa 5ta edición. madrid-españa. 590 pp.

NAMESNY, A. 1996. Pimientos. Ediciones de horticultura, sl. Madrid-españa. 135 pp.

NUEZ, F. GIL ORTEGA,R. COSTA, J. 1996. El cultivo de pimientos, chiles y ajies. Ediciones Mundiprensa Madrid-España. 586 pp.

OLIVEIRA, T. Y COLS. 2002. Efeito de antocianina e própolis em diabetes induzida em coelhos / Effects of anthocyanin and propolis in diabetic rabbits. Medicina (Ribeirão Preto);35(4):464-469, out.dez.. tab.

PETOSEED. 1986. Catalogo de cultivares. 72 pp. EEUU.

PETOSEED. Cultivo de pimiento al aire libre. 4 pp. sin fecha. Chile.

RAMÍREZ, F. 2000. Manejo nutricional y fertilización balanceada en el cultivo de páprika. Manejo del cultivo de páprika. Arequipa. 
SALINAS MORENO, ET AL. 2005. Extracción y uso de pigmentos del grano de maíz (zea mays L) como colorantes en yogurt / Extraction and use of pigments from maize grains (zea mays L) as colorants in yogur. Archv. Latinoam. Nutr. 55(3):293-298, sept.

SOLANO M.R. 1999. Efecto de la Fertirrigación N-P$\mathrm{K}$ en el Rendimiento y el contenido de Antocianinas de tres variedades de Maíz Morado (Zea mays L.) bajo R.L.A.F.: Goteo. Tesis. UNA. Lima.
VADEMÉCUM AGRARIO. 1999-2000. Ediprensa. Lima-Perú. 137 pp.

VILLARIVAU, A Y GONZALES, J. 1999. Planteles, semilleros, viveros. ediciones de horticultura, sl.. Madrid-España. 271 pp.

ZAPATA, M. BAÑON, S Y CABRERA, P. 1992. El pimiento para pimentón. Ed. mundi-prensa madrid-españa. $240 \mathrm{pp}$.

Tabla 1: Variación de los niveles de Triglicéridos (mg/dL) del grupo experimental con una dieta suplementada con chicha morada con el $20 \%$.

\begin{tabular}{lcccc}
\hline Consumo & $\mathbf{N}^{\mathbf{0}}$ Rata & Basal & $\begin{array}{c}\text { Día 15 } \\
\text { Dieta } \\
\text { hipergrasa }\end{array}$ & $\begin{array}{c}\text { Día 30 } \\
\text { Dieta } \\
\text { experimental }\end{array}$ \\
\hline & 1 & 44.88 & 181.90 & 49.30 \\
Chicha & 2 & 42.08 & 179.70 & 47.04 \\
Morada & 3 & 45.18 & 182.60 & 49.98 \\
& 4 & 41.78 & 179.00 & 46.36 \\
& 5 & 44.78 & 182.00 & 49.89 \\
\hline Promedio & 6 & 42.18 & 179.60 & 46.45 \\
D.S & & 43.48 & 180.80 & 48.17 \\
\hline
\end{tabular}

Tabla 2: Variación de los niveles de Triglicéridos ( $\mathrm{mg} / \mathrm{dl}$ ) del grupo experimental con una dieta suplementada con antocianinas al 5\%.

\begin{tabular}{lcccc}
\hline Consumo & $\mathbf{N}^{\mathbf{0}}$ Rata & Basal & $\begin{array}{c}\text { Día 15 } \\
\text { Dieta } \\
\text { hipergrasa }\end{array}$ & $\begin{array}{c}\text { Día 30 } \\
\text { Dieta } \\
\text { experimental }\end{array}$ \\
\hline & 7 & 42.18 & 179.60 & 27.79 \\
Antocianina & 8 & 44.83 & 182.15 & 24.93 \\
& 10 & 42.13 & 179.45 & 28.08 \\
& 11 & 45.14 & 182.40 & 24.64 \\
& 12 & 43.48 & 179.20 & 27.74 \\
Promedio & & 43.26 & 180.80 & 24.98 \\
D.S. & & 1.45 & 1.41 & 26.36 \\
\hline
\end{tabular}


Tabla 3: Variación de los niveles de Colesterol ( $\mathrm{mg} / \mathrm{dL})$ del grupo experimental con una dieta suplementada con chicha morada con el $20 \%$.

\begin{tabular}{ccccc}
\hline Consumo & No Rata & Basal & $\begin{array}{c}\text { Día 15 } \\
\text { Dieta } \\
\text { hipergrasa }\end{array}$ & $\begin{array}{c}\text { Día 30 } \\
\text { Dieta } \\
\text { experimental }\end{array}$ \\
\hline & 1 & 38.01 & 97.76 & 36.51 \\
Chicha Morada & 2 & 35.81 & 98.48 & 34.25 \\
& 3 & 38.71 & 95.56 & 37.19 \\
& 4 & 35.11 & 98.01 & 33.57 \\
& 5 & 38.11 & 94.86 & 36.6 \\
\hline Promedio & 6 & 35.71 & 95.31 & 34.16 \\
\hline D.S. & & 36.91 & 96.66 & 35.35 \\
\hline
\end{tabular}

Tabla 4: Variación de los niveles de Colesterol (mg/dL) del grupo experimental con una dieta suplementada con antocianinas al 5\%.

\begin{tabular}{lcccc}
\hline Consumo & No $^{\mathbf{0}}$ Rata & Basal & $\begin{array}{c}\text { Día 15 } \\
\text { Dieta } \\
\text { hipergrasa }\end{array}$ & $\begin{array}{c}\text { Día 30 } \\
\text { Dieta } \\
\text { experimental }\end{array}$ \\
\hline & 7 & 35.71 & 95.31 & 30.35 \\
Antocianina & 8 & 38.26 & 97.86 & 28.09 \\
& 10 & 35.56 & 98.26 & 31.03 \\
& 11 & 38.51 & 95.46 & 27.41 \\
& 12 & 36.31 & 95.06 & 30.44 \\
Promedio & & 36.71 & 96.66 & 28.00 \\
D.S. & & 1.41 & 96.43 & 29.22 \\
\hline
\end{tabular}

Tabla 5: Variación de los niveles de Colesterol LDL (colesterol malo) (mg/dL) del grupo experimental con una dieta suplementada con chicha morada con el $20 \%$.

\begin{tabular}{ccccc}
\hline Consumo & No Rata & Basal & $\begin{array}{c}\text { Día 15 } \\
\text { Dieta } \\
\text { hipergrasa }\end{array}$ & $\begin{array}{c}\text { Día 30 } \\
\text { Dieta } \\
\text { experimental }\end{array}$ \\
\hline & 1 & 4.64 & 25.05 & 4.21 \\
Chicha Morada & 2 & 5.34 & 22.19 & 1.95 \\
& 3 & 1.74 & 25.34 & 4.89 \\
& 4 & 2.44 & 21.9 & 1.27 \\
\hline Promedio & 5 & 2.34 & 25 & 4.3 \\
D.S. & 6 & 4.89 & 22.24 & 1.86 \\
\cline { 2 - 5 } & & 3.57 & 23.62 & 3.08 \\
& & 1.56 & 1.66 & \\
\hline
\end{tabular}


Tabla 6: Variación de los niveles de Colesterol LDL (colesterol malo) (mg/dl) del grupo experimental con una dieta suplementada con antocianinas al $5 \%$.

\begin{tabular}{lcccc}
\hline Consumo & No $^{\circ}$ Rata & Basal & $\begin{array}{c}\text { Día 15 } \\
\text { Dieta } \\
\text { hipergrasa }\end{array}$ & $\begin{array}{c}\text { Día 30 } \\
\text { Dieta } \\
\text { experimental }\end{array}$ \\
\hline & 7 & 4.89 & 22.24 & 1.43 \\
Antocianina & 8 & 5.14 & 24.97 & 1.13 \\
& 10 & 4.74 & 22.27 & 1.5 \\
& 11 & 1.94 & 25.28 & 1.06 \\
& 12 & 3.54 & 23.62 & 1.59 \\
Promedio & & 3.74 & 23.39 & 0.97 \\
D.S. & & 1.41 & 1.47 & 1.28 \\
\hline
\end{tabular}

